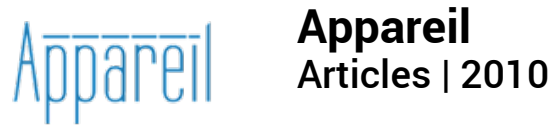

\section{Le bocal de Veyne}

Alain Brossat

\section{OpenEdition \\ Journals}

\section{Édition électronique}

URL : http://journals.openedition.org/appareil/1140

DOI : 10.4000/appareil. 1140

ISSN : 2101-0714

\section{Éditeur}

MSH Paris Nord

\section{Référence électronique}

Alain Brossat, « Le bocal de Veyne », Appareil [En ligne], Articles, mis en ligne le 27 décembre 2010, consulté le 30 juillet 2020. URL : http://journals.openedition.org/appareil/1140 ; DOI : https://doi.org/ 10.4000/appareil.1140

Ce document a été généré automatiquement le 30 juillet 2020.

\section{(c) (i) (9)}

Appareil est mis à disposition selon les termes de la Licence Creative Commons Attribution - Pas d'Utilisation Commerciale - Pas de Modification 4.0 International. 


\title{
Le bocal de Veyne
}

\author{
Alain Brossat
}

$1 \mathrm{Au}$ fond, l'acharnement quelque peu compulsif avec lequel nous sommes aujourd'hui portés à affirmer que toute forme de vie doit être en mesure de faire valoir ses droits et être protégée est l'écran qui nous masque un terrible secret: ce n'est que d'hier, à peine, que nous sommes devenus si « vertueux » et sensibles, en la matière, au point de nous scandaliser des images violentes de gavage des oies et canards et de renoncer, au nom du droit à la vie de ces malheureux animaux, à consommer du foie gras. Hier encore, nous étions tous plutôt de ce bois dont est fait un personnage comme ce propriétaire de troupeaux énergique et borné incarné par John Wayne dans La rivière rouge: portés tout naturellement à considérer un troupeau comme de la viande sur pied, donc un capital, destiné à faire du «good beef» et à «make men strong» - rien d'autre'.

2 Ici encore, les brutales ruptures de niveau qui se manifestent dans l'ordre des discours, des représentations et des sensibilités sont, pour nous, extraordinairement éprouvantes. Comme aime à le dire Paul Veyne, nous pensons et parlons à l'intérieur d'un bocal aux parois transparentes, donc nous ne voyons pas le bocal lui-même, nous n'avons pas conscience de la façon dont il enferme tous nos discours, nous nous étonnons seulement (nous amusons, nous scandalisons...) des énoncés que d'autres ont pu former dans d'autres bocaux - et quant à la question de savoir comment l'on passe d'un bocal à l'autre - nous n'en avons pas la moindre idée ${ }^{2}$. C'est précisément la raison pour laquelle se manifeste cette propension si vive à rétablir de la "pensée magique " dès lors que nous avons à franchir ce pont aux ânes épistémologique - comment se produit l'opération du passage d'un ordre de discours à un autre, d'un régime d'énonciation du vrai à un autre?

3 Voyons cela. D'un jour sur l'autre, pratiquement, Alexis Carrel, prix Nobel de médecine en 1912 pour ses expériences sur les tissus et les vaisseaux sanguins, s'est transformé de héros de la science et figure éminente de l'humanisme contemporain en esprit maléfique ayant part liée avec toutes les horreurs de la première moitié $\mathrm{du} \mathrm{xx}^{\mathrm{e}} \mathrm{siècle} \mathrm{-}$ ceci vers la fin des années 1980 . On s'est mis à débaptiser force rues, écoles et piscines portant son nom et on s'est avisé que ce best-seller absolu qui circulait de main en 
main, de génération en génération, depuis les années 1930 - L’Homme cet inconnu n'était rien d'autre qu'une sorte d'écrit nazi propageant le pire de l'eugénisme négatif ${ }^{3}$. D'un jour sur l'autre, en effet, une petite phrase passée inaperçue aux yeux de milliers et de milliers de lecteurs a brusquement sauté d'entre les pages comme un diable et s'est mise à brûler la rétine des contemporains ; une phrase qui disait qu'une politique responsable d'amélioration de la qualité de la «race» française, une politique hygiéniste et sanitaire conséquente et moderne, supposait que l'on affronte sans faiblesse la question de la vie humaine déficiente, de mauvaise qualité, susceptible d'affaiblir la « race » - le problème des débiles mentaux, de ceux qui souffrent de tares héréditaires et qui sont susceptibles de contribuer à la dégénérescence de la population dans notre pays ; ceux-là, statuait Carrel, comme en passant, il faudrait avoir le courage de les "éliminer", sur un mode bien sûr humanitaire et indolore - par le "gaz", précisait-il encore - et c'est là, précisément, que prit racine sa déchéance posthume. Le crime rétrospectif, rétroactif de Carrel, inspirateur direct au demeurant de toutes sortes de savants respectés dont l'étoile, elle, n'a pas pâli, comme Jean Rostand, est distinct : il pense, en savant, en publiciste, en bio-politicien, si l'on veut, il pense ces questions à l'intérieur d'un bocal que nous avons résolument rangé sur l'étagère des horreurs du passé ; il pense ces questions aux conditions de l'énoncé mis en forme par le « grand discours » (pas seulement une doctrine) de l'eugénisme : l'amélioration de la qualité du vivant humain passe non seulement par le développement d'une politique d'encadrement sanitaire renforcée, de développement de l'hygiène et des vaccinations, elle passe aussi par le combat énergique contre les facteurs de dégénérescence, notamment par la stérilisation des sujets tarés, voire la "mise à l'écart » de ces catégories que l'on peut identifier comme de qualité déficiente, irrécupérables de ce point de vue, des catégories qu'un certain intégriste eugéniste (l'eugénisme négatif) ira jusqu'à décréter « indignes de vivre».

4 Quoi qu'il en soit, Carrel, dans L'Homme cet inconnu, ne fait jamais que livrer une version un peu musclée du programme fondamental selon lequel le «faire vivre» de la partie saine de la population passe par des opérations de sélection et de partage, susceptibles de prendre des formes infiniment variables ${ }^{4}$. Et, « un jour », cette vision des choses et les énoncés qui en répondent deviennent odieux, insupportables, ils portent la marque irrécusable du barbare. Mais comme nous sommes incapables de rendre compte de cette opération par laquelle nous passons d'un bocal à un autre, il nous faut organiser toutes sortes de rites et d'exorcismes par lesquels nous allons célébrer, si l'on peut dire, notre établissement dans le nouveau bocal et nous séparer de tout ce qui peut rappeler l'ancien - les discours et opérations de séparation du vrai d'avec le faux qui y prévalaient. Des rites et des exorcismes destinés, notamment, à effacer la blessure narcissique que ce genre de saut d'un bocal à l'autre inflige à notre continuisme, notre philosophie spontanée du progrès, notre rationalisme. La diabolisation de Carrel est ici bien commode, car elle nous permet de nous débarrasser de l'encombrant problème des discontinuités radicales, en matière d'ordre des discours, et donc de l'épineuse question de la relativité de nos énoncés et régimes de vérités à un topos singulier - la question de l'historicité de nos discours et de leur inscription dans l'horizon du vrai et du faux, du juste et de l'injuste. La question horriblement incommode, donc, de la relation qui s'établit entre l'opération de séparation du vrai et du faux et des conditions singulières d'historicité. Ce n'est pas pour rien que Foucault conçut un jour le projet de s'atteler à une « histoire de la vérité »... 
5 Ce que dit Carrel fait moins de lui un double satanique du Dr Mengele qu'un zélé fonctionnaire de la vérité enfermé dans son bocal (qui n'est pas tout à fait la même chose que "son temps »). La petite phrase à retardement qui met le feu aux poudres un demi-siècle après sa publication perd beaucoup de son poison propre si on la met en relation avec ce qui fut une pratique routinière durant la seconde guerre mondiale en France : l'abandon des malades mentaux enfermés dans les asiles et considérés pour la plupart comme incurables, comme du déchet humain, à une mort lente, dans les asiles. Morts de faim, de cachexie ${ }^{5}$. Ce qui singularise les nazis, parmi les nombreux acteurs de la politique de séparation du vivant de qualité d'avec le vivant indigne de vivre, c'est leur fanatisme d'entrepreneurs des opérations de hiérarchisation, d'étiquetage et de partage. Mais la «philosophie de la vie» qui statue que le «faire vivre » des uns suppose la mise à l'écart des autres, selon des modalités pouvant aller de l'abandon plus ou moins concerté au "faire mourir » industriel, ils la partagent avec quantité de poissons et batraciens moins patibulaires, locataires du même bocal - notamment parmi les élites scientifiques.

6 L'ordre des discours « corrects » sur ce sujet est balisé, d'un côté par les horreurs extrêmes du programme T4 et les camions à gaz, certes, mais, de l'autre, par la banalité d'un discours "autorisé " dont voici un échantillon : ces réflexions de sens commun formulées par Édouard Toulouse, une sommité médicale de la fin du XIX ${ }^{\mathrm{eo}}$ siècle : «Le séjour de tous ces chroniques [les malades mentaux enfermés à vie dans les asiles] inoffensifs dans nos asiles où la vie est si dispendieuse est presque une extravagance d'assistance, ainsi que l'a écrit un aliéniste anglais. Pourquoi tant dépenser pour cultiver en serre chaude et prolonger indéfiniment l'existence d'un si grand nombre d'idiots et de déments ? Les uns n'ont jamais pu donner, les autres ne pourront jamais plus donner aucun profit à la société. Cette dernière doit les assister congrûment [c'est moi qui souligne] et réserver le reste de son argent pour les malades aigus et pour tant d'autres infortunés, par exemple les enfants abandonnés, qui sont un capital certain dont les revenus dépasseront tous les sacrifices faits pour eux " (Rapport sur l'existence des aliénés en France et en Écosse, Conseil général de la Seine, 1898) ${ }^{6}$.

7 La virulence avec laquelle nous entreprenons, à la fin des années 1980, de nous séparer d'Alexis Carrel, en le brûlant en effigie, vise à surmonter (sur un mode tant soit peu «magique ", une fois encore) le sentiment d'incrédulité horrifiée que nous inspire notre extrême proximité avec ce passé : celui où prévalait encore un régime de discours ayant «la vie» pour objet, un régime selon lequel s'imposait encore l'évidence des partages entre ce dont il convenait d'améliorer le « faire vivre » et ce qui pouvait être abandonné à la mort (ou devait être voué à la mort). Le précipice qu'il est bien difficile de contempler sans succomber au vertige est donc celui-ci : dans le premier âge de la biopolitique, s'impose cette approche de « la vie » (entée sur des modes discursifs et des représentations traditionnels) selon laquelle la prise en charge de la vie ne peut être que sélective et fondée sur des opérations de hiérarchisation; cette règle ou ce régime nous sont devenus insupportables dans la mesure où ils supposent des "décrets » statuant sur le « déchet » abject ou l'en-trop du vivant humain et sur le tri entre le bon grain et l'ivraie de ce même vivant. En tant, en effet, que notre "époque » (au sens de "ce qui fait époque ») est celle qui nous établit dans la position du «témoin d'Auschwitz ", c'est-à-dire de la catastrophe à laquelle conduit, pensons-nous, ce mode de problématisation de « la vie » par la première modernité biopolitique, nous allons rejeter avec horreur et indignation toute notion d'un tri bénéfique, d'une hiérarchie 
légitime, d'un partage organisateur parmi les formes du vivant - humain dans un premier temps, vivant tout court selon la dynamique propre aux nouveaux énoncés qui acquièrent ici force de loi. Nous allons "passer sur l'autre bord", là où va s'imposer l'évidence du « droit à la vie ».

8 «Pendant des années et des années, écrit Péguy dans Clio, pendant dix, quinze, vingt ans, pendant trente ans vous vous acharnez à un certain problème et vous ne pouvez apporter aucune solution et vous vous acharnez à un certain mal et vous ne pouvez apporter aucun remède. Et tout un peuple s'acharne. Et des générations entières s'acharnent. Et tout d'un coup, on tourne le dos [c'est moi qui souligne, A. B.]. Et le monde entier a changé de face. Ni les mêmes problèmes ne se posent plus (il s'en posera assez d'autres), ni les mêmes difficultés ne se présentent, ni les mêmes maladies ne sont plus considérables. Il n'y a rien eu. Et tout est autre [c'est moi qui souligne, A. B.]. Et tout est nouveau. Il n'y a rien eu. Et tout l'ancien n'existe plus et tout l'ancien est devenu étranger.

Et on ne sait plus de quoi on parlait [...] [c'est moi qui souligne, A. B.]. »7

10 La seconde modernité biopolitique sera celle qui établit le postulat fondateur de l'indistinction entre les formes de vie, « toute vie » proclamant son « droit » et devant à ce titre être protégée. C'est la raison pour laquelle, dans ce nouvel ordre des discours, des notions comme celle de "race» (instrument d'opérations de hiérarchisation brutales entre différents groupes humains), de " dégénérescence », de "sang » (au sens biopolitique) ont subi un violent discrédit ${ }^{8}$.

11 D'une façon toujours plus contraignante, nous allons problématiser notre propre condition comme civilisée, "humaine ", en tant qu'elle fonde sa légitimité sur ce postulat de sanctuarisation (qui n'est pas équivalente ici à sacralisation) de toute forme de vie humaine. Pour jouer pleinement son rôle en termes de production d'identité ou d'inscription positive dans l'actualité, ce postulat devrait pouvoir se présenter comme validé par une longue tradition et inscrit dans une longue durée - or, il n'en est rien, il est, tout au contraire, une sorte de dernier cri, brusquement surgi dans l'après-coup du méta-traumatisme nommé Auschwitz.

12 S'il est une chose dont aucune espèce d'approche historiciste ou positiviste de la formation des récits et des discours sur le passé (mais aussi, sur «la vie») ne puisse rendre compte, c'est bien de phénomènes absolument déroutants comme l'absence d'une ligne, d'un mot évoquant ce qui, pour nous, est le fait marquant de l'époque, celui qui surplombe tous nos discours sur le passé récent et la vie aussi bien - la Shoah, donc - dans tel manuel scolaire consacré dans les années1960 au monde contemporain et publié sous la houlette de sommités de la discipline historique contemporaine ou, aussi bien, dans tel classique de la philosophie morale de l'après-guerre'.

13 En effet, s'il s'agit de faire référence à l'ancienneté des discours, ce qui frapperait, une fois encore, ce serait bien la façon dont nos systèmes d'évidences contemporains (sur ces questions de "la vie») se construisent tout entiers au rebours des certitudes et routines discursives anciennes. Dieu sait (façon de parler) à quel point l'impératif de la "protection de la vie » a dû devenir catégorique et être placé hors de portée de toute discussion pour que l'action des aventuriers de l'Arche de Zoé, au Tchad, fin 2007, début 2008, puisse apparaître comme une entreprise vertueuse mais maladroite de sauvetage de vie fragile, plutôt que comme un acte de brigandage néo-colonialiste, vénal et cynique. C'est que l'enfance est devenue, par excellence, cette forme de la vie qui se doit d'être protégée, envers et contre tout et que toute action, aussi litigieuse fût-elle, 
qui fait recours à un tel alibi tend à devenir, par pur automatisme, hautement louable...

Pourtant, Rousseau, fondateur, nous serine-t-on, de l'éducation moderne et grand ami de l'enfance, ne voyait pas du tout les choses ainsi : parmi les enfants auxquels il s'intéressera pour les faire profiter de ses principes éducatifs révolutionnaires, il opère d'emblée un tri impérieux: "Je ne me chargerais pas d'un enfant maladif et cacochyme, dût-il vivre quatre-vingts ans. Je ne veux point d'un élève toujours inutile à lui-même et aux autres, qui s'occupe uniquement à se conserver, et dont le corps nuise à l'éducation de l'âme. Que ferais-je en lui prodiguant vainement mes soins, sinon doubler la perte de la société et lui ôter deux hommes pour un? Qu'un autre à mon défaut se charge de cet infirme, j'y consens, et j'approuve sa charité ; mais mon talent à moi n'est pas celui-là : je ne sais point apprendre à vivre à qui ne songe qu'à s'empêcher de mourir [c'est moi qui souligne] $»^{11}$.

Pour Rousseau, en effet, vivre ne saurait être équivalent à "se conserver ", vivre ne prend sens et ne s'inscrit dans un horizon axiologique que pour autant que cela consiste à affronter le négatif - à commencer par l'exposition à la mort, la maladie et la douleur. Un enfant ne deviendra donc un vivant qualifié (dont la vie ait une valeur et qui soit capable de l'affirmer comme singularité) que pour autant qu'il aura appris à affronter la fragilité de sa condition et sa constante exposition au risque de la mort: «Faute de savoir se guérir, que l'enfant sache être malade : cet art supplée à l'autre [la médecine], et souvent réussit beaucoup mieux ; c'est l'art de la nature. Quand l'animal est malade, il souffre en silence et se tient coi; or on ne voit pas plus d'animaux languissants que d'hommes ${ }^{12}$. Aussi bien, insiste Rousseau sans ménagement, la notion d'une valeur intrinsèque de chaque vie humaine commençante, laquelle impliquerait la nécessité d'une prise en charge active et constante de toutes, n'a pas de sens: en effet, "des enfants qui naissent, la moitié, tout au plus, parvient à l'adolescence; et il est probable que votre élève n'atteindra pas l'âge d'homme... ${ }^{13}$. Une remarque qui suffit à nous rappeler les conditions matérielles, sanitaires, environnementales qui forment l'a priori nécessaire de notre propre philosophie du « droit à la vie ».

16 L'hostilité persistante que Rousseau manifeste à l'endroit de la médecine, art faux et artificieux pour lui, tient à son attachement à ce principe (qui installe sa philosophie de «la vie» aux antipodes de celle qui prévaut en notre époque): la vie de chacun des vivants est, pour l'essentiel entre les mains de la nature et elle doit le rester; c'est en affrontant avec ses propres forces, avec son propre courage et sa propre énergie ce qui menace son intégrité, sa santé, sa vie, qu'un individu devient « un homme » présentant une qualité véritablement humaine ; c'est donc mettre le doigt dans un bien dangereux engrenage que de confier le soin de sa santé et de sa vie à la médecine: "Je ne sais, pour moi, de quelle maladie nous guérissent les médecins, mais je sais qu'ils nous en donnent de bien funestes : la lâcheté, la pusillanimité, la crédulité, la terreur de la mort : s'ils guérissent le corps, ils tuent le courage [...] C'est l'amusement des gens oisifs et désœuvrés qui, ne sachant que faire de leur temps, le passent à se conserver [...] Il faut à ces gens-là des médecins qui les menacent pour les flatter et qui leur donnent chaque jour le seul plaisir dont ils soient susceptibles, celui de n'être pas morts. $»^{14}$

La vie, donc, comme vie vécue, se doit de se maintenir au plus près de la nature, sans que des interventions artificieuses, comme le sont le plus souvent celles des médecins, ne viennent perturber ce cours naturel des choses. Pour l'essentiel, dit Rousseau, «la 
nature a, pour fortifier le corps et le faire croître, des moyens qu'on ne doit jamais contrarier ». Mais le libre jeu des forces naturelles n'exclut, évidemment, ni la maladie, ni la douleur, ni le risque de la mort prématurée. Ces possibilités, l'homme doit les envisager et les accepter comme inhérentes à sa condition. Mieux, elles sont inséparables de son statut moral, de ce qui va le faire passer de la condition de vivant simple à celle de sujet moral: "L'homme qui ne connaitrait pas la douleur, ne connaîtrait ni l'attendrissement de l'humanité, ni la douceur de la commisération; son cœur ne serait ému de rien, il ne serait pas sociable, il serait un monstre parmi ses semblables $»^{15}$.

Insensibilisation physique, immunisation contre la douleur, et insensibilisation morale, indifférence croissante au malheur d'autrui vont donc de pair ; Rousseau voit ici loin vers l'avant, en décrivant au fond par anticipation le processus de la civilisation de la modernité occidentale comme un procès d'insensibilisation générale. Au reste, la sagesse dont il se fait l'avocat consiste à exhorter les hommes à accepter pleinement leur condition de mortalité, dans tous ses aspects, pour autant que celle-ci est une condition naturelle, et à réinterpréter ce qui, en première approche, apparaît comme un indice d'imperfection, un trait de fragilité, comme le moyen par lequel l'humain peut affirmer sa qualité propre, développer ses potentialités morales et, finalement, accéder au bonheur - «Si l'on nous offrait l'immortalité sur la terre, qui est-ce qui voudrait accepter ce triste présent ? $»^{16}$. Mortalité, finitude de l'homme, imperfection, condition d'exposition et susceptibilité d'être affecté propres à l'humain sont donc pour Rousseau les facteurs qui sont établis au fondement d'une valeur propre et singulière de l'être humain. Ce qui signifie que, dans cette perspective, des différenciations se produisent au sein du vivant, entre le vivant humain et le vivant animal notamment, mais à l'intérieur du vivant humain lui-même aussi: des puissances de vie différenciées se manifestent, des «chances» de vie aussi, car tel est l'état des choses naturelles, intangible et immuable. La référence à ces prétendus invariants va, bien sûr, subir un vigoureux démenti dans tout le cours de notre modernité, puisque l'on y assistera au déplacement incessant de la ligne de partage entre ce qui est, en matière de prise en charge du vivant (par nous-mêmes), abandonné au cours des choses (à la nature ou « au destin", si l'on veut) et ce sur quoi nous allons exercer une action régulatrice, transformatrice, nous assurer des prises, voire assurer une maîtrise. Si bien que la charge de Rousseau contre la médecine « utile à quelques hommes, mais funeste au genre humain » suscitera aujourd'hui comme un sentiment d'incrédulité amusée. Mais, d'un autre côté, c'est précisément cette sorte de candeur anti-moderne, ce parti quasi-« diogénique » en faveur de la vie naturelle contre la civilisation et ses artéfacts (incluant les savoirs et les pratiques de la médecine comme les attraits des spectacles) qui la dotent de cette qualité de voyance grâce à laquelle il décrit par avance cette pente subjective $\mathrm{du}$ procès de modernisation: insensibilisation, immunisation, sanctuarisation indistincte du vivant.

Rousseau nous aide à comprendre la difficulté que nous éprouvons à remettre nos pas dans ceux d'une sensibilité, toute proche encore, mais antérieure au «droit à la vie » entendu comme règlement général des sensibilités, norme des pensées et des énoncés corrects. Que des enfants meurent, dit-il, et en nombre, c'est dans l'ordre des choses; par conséquent, n'ayons garde de nous attacher trop tôt aux enfants, ni à tous les enfants, notamment aux enfants maladifs et geignards, bref ceux dont l'énergie vitale apparaît déficiente. Proposition infiniment choquante pour nous qui, en langue 
nietzschéenne, sommes passés du côté des faibles et des victimes - mais dont l'écho s'entend pourtant si couramment en ce « monde d'avant »...

\section{NOTES}

1. La rivière rouge, film de Howard Hawks, avec John Wayne, Montgomery Clift... (1948).

2. Paul Veyne: Comment on écrit l'histoire suivi de Foucault révolutionne l'histoire, Point Seuil « Histoire », 1971. Également: Michel Foucault, sa pensée, sa personne, Albin Michel, 2008.

3. Alexis Carrel : L'homme cet inconnu, Plon, 1935.

4. L'eugénisme négatif, avant d'être érigé en doctrine par les nazis, a connu de beaux jours dans les pays anglo-saxons et scandinaves. Voir à ce propos Daniel J. Kevles: Au nom de l'eugénisme génétique et politique dans le monde anglo-saxon, PUF 1995 et André Pichot : La société pure - de Darwin à Hitler, Flammarion, 2000.

5. Voir à ce propos Max Lafont : L'extermination douce, Le Bord de l'eau, Latresne, 2000.

6. Robert Castel : La gestion des risques, Minuit, 1981, p. 80.

7. Charles Péguy, Clio op. cit. supra. p. 266.

8. Voir à ce propos Drôle d'Époque $\mathrm{n}^{\circ} 19$, automne 2006, dossier « Figures politiques du sang ».

9. Le monde contemporain, manuel d'histoire destiné aux classes terminales, publié sous la direction de L. Genet, R. Rémond, P. Chaunu, A. Marcet, J. Ki-Zerbo, Hatier 1962. Etienne Borne : Le problème du mal, PUF, 1958.

10. Je me permets de renvoyer sur ce point à mon article in Raison présente $n^{\circ} 166$ «Les perversions du pastorat démocratique ».

11. Émile ou de l'éducation, Garnier, 1961, premier livre, p. 29.

12. Ibid., premier livre, p. 31.

13. Ibid., premier livre p. 61.

14. Ibid., livre premier p. 29.

15. Ibid., second livre, p. 73.

16. Ibid., second livre, p. 65 .

\section{RÉSUMÉS}

Nous formons nos pensées, énonçons des jugements et parlons, remarque Paul Veyne, de l'intérieur d'un bocal aux parois transparentes dont nous ignorons l'existence. Aussi éprouvonsnous une grande incrédulité face aux conditions différentes dans lesquelles d'autres, enfermés dans d'autres bocaux, forment leurs pensées, énoncent des jugements, parlent. Nous passons d'un bocal à l'autre, parfois, mais subrepticement, sans nous aviser que les conditions qui rendent possibles nos pratiques du vrai et du faux ont changé. Nous sommes de bien étourdis poissons rouges ou batraciens... 
Cet article présente quelques unes des conséquences de cette proposition, appliquée à la façon dont nous envisageons la question du «droit à la vie » aujourd'hui, dont nous problématisons l'immunisation nécessaire, sans restriction ni exclusion, du vivant humain - et au delà, de toute forme de vie. Il rappelle combien cette approche s'est récemment imposée à nous et contraste avec toutes sortes d'énoncés qui, hier encore, faisaient autorité...

INDEX

Mots-clés : bocal, droit à la vie, enfance, eugénisme, médecine, ordre des discours, Paul Veyne 